\title{
Recent advances in skin collagen: functionality and non-medical applications
}

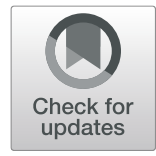

Yanting $\mathrm{Han}^{1}$, Jinlian $\mathrm{Hu}^{2^{*}}$ and Gang Sun ${ }^{3^{*}}$

\begin{abstract}
During nature evolution process, living organisms have gradually adapted to the environment and been adept in synthesizing high performance structural materials at mild conditions by using fairly simple building elements. The skin, as the largest organ of animals, is such a representative example. Conferred by its intricate organization where collagen fibers are arranged in a randomly interwoven network, skin collagen (SC), defined as a biomass derived from skin by removing non-collagen components displays remarkable performance with combinations of mechanical properties, chemical-reactivity and biocompatibility, which far surpasses those of synthetic materials. At present, the application of SC in medical field has been largely studied, and there have been many reviews summarizing these efforts. However, the generalized view on the aspects of SC as smart materials in non-medical fields is still lacking, although SC has shown great potential in terms of its intrinsic properties and functionality. Hence, this review will provide a comprehensive summary that integrated the recent advances in SC, including its preparation method, structure, reactivity, and functionality, as well as applications, particularly in the promising area of smart materials.
\end{abstract}

\section{Introduction}

Living organisms have ingeniously developed hierarchical structures to create high-performance biological materials from a fairly limited choice of elements and compounds during long-term evolution and selection. The protein, composed of amino acids, is such typical materials. General, characteristic conformation of proteins includes $\alpha$ helix, $\beta$ sheet, triple helix and random coil [1]. These structures can exist in biological tissues either alone or simultaneously to perform specific functions. Although many efforts have been devoted to synthesizing materials that mimic natural structures, their target performance are rarely comparable to natural ones. Hence, the direct translation of natural motifs into functional materials may offer a spectrum of facile pathways towards unprecedented properties that are favorable for practical uses in a variety of engineering and industry fields. It should be addressed that animal skin, readily available in large quantity as

\footnotetext{
* Correspondence: jinliahu@cityu.edu.hk; gysun@ucdavis.edu

${ }^{2}$ Department of Biomedical Engineering, City University of Hong Kong, Kowloon, Hong Kong, China

${ }^{3}$ Division of Textiles and Clothing, University of California, Davis, Davis, USA Full list of author information is available at the end of the article
}

byproduct of meat industry can be regarded as a sophisticated research object due to its structural component of collagen and unique fibrous network.

In the skin, the intricated collagen fiber bundles, which has a hierarchical structure built up a 3D fibrious network. This structure is difficult for today's synthetic material to imitate in terms of composition and structure. This pure skin collagen (SC) matrix can be obtained by removing epithelial components (keratinocytes, sweat glands and sebaceous glands) and dermal non-structural components (fibroblasts, vascular endothelium and smooth muscle, etc.). The unique fibrous features, mechanical strength and flexibility make the SC preferable material for traditional product such as leather. Although this industry has brought value to this by-product, the deep study and advanced utilization is very limited. In addition, With increasingly stringent environmental regulations for products originated from natural source, materials based on biomass including collagen [2] have attracted attention in the materials field. Particularly, the emerging smart materials need fresh blood. Hence, as a renewable resource, SC has a lot of potential to be developed. Particularly, the collagen has excellent biological (e.g. biocompatibility, low antigenicity 
and biodegradability) and physical properties (e.g. mechanical strength, flexibility, permeability, structural stability) [3, 4], which leads SC as desirable materials in medical fields where biocompatibility and mechanical flexibility are essential. Besides, the existence of metal chelates functional groups (eg. - $\mathrm{OH},-\mathrm{COO}$ ) on collagen provides the feasibility of functionalization of $\mathrm{SC}$ for extended applications. Under constant research in recent years, the hidden smart functions of SC have been gradually revealed, which is a significant inspiration to research filed of natural material.

Briefly, the mainly functionalization and applications of SC can be divided into two categories: one is based on its biological properties including its good biocompatibility, low antigenicity and biodegradability, which is suitable for cosmetic [5], biomedical materials [6], tissue engineering materials [7] and drug release fields [8]; Then another one contains other studies of SC in nonmedical fields. To date, the application of SC in the medical field has been largely studied, and there have been many reviews summarizing these efforts. However, the generalized view on the aspects of SC as functional and smart materials in non-medical fields is still lacking. Hence, this review will be focused on recent progress on structural study, functionalization and non-medical applications of SC. Based on its fibrous matrix features and performance, such as unique hierarchical structure, good mechanical properties and highly chemical reactivity, SC is regarded as preferable material for covering, adsorbing and electronic skin, etc. It should be noted that the intrinsic water responsive shape memory of SC has recently been revealed recently, which indicating a new development direction of SC. Additionally, in this review, the perspective of study of SC has also been given.

\section{Preparation of SC}

From a biological anatomical point of view, the skin is composed of two major layers: a superficial epidermis and a dermis [9]. During the preparation process of SC, the epidermis would be removed, hence, the obtained SC mainly reserves the distinguished structure of dermis. The reticular layer of dermis is composed of thick collagen fiber bundles which are intertwined into a dense tissue. This layer generally contains little sweat glands, fatty glands, hair follicles, and there is fewer elastic fibers and grease. That means the reticular layer appears ideal reticulated (net-like) three-dimensional structure due to a tight meshwork of fibers.

Based on the physicochemical properties of each components in skin, methods employed for producing SC from fresh animal skin including treatment with alkali, surfactant, and enzymes. Briefly, the process can be summarized into several key steps (Fig. 1a): degrease, unhairing, alkali/acid enzyme hydrolysis and final wash
$[10,11]$. In consideration of environmental protection issues, enzymes are preferred to be used instead of alkali in the hair removal process. Dettmer et al. [12] described the use of an enzyme produced by a new strain of Bacillus subtilis for unhairing. This enzymatic preparation lead to the COD reductions of $42 \%, 100 \%$, and $82 \%$ compared with chemical process, and save more than $50 \%$ time for unhairing process. Rose et al. developed a lime- and sulfide-free unhairing of skins using animal and/or herbal (plant) enzymes. This unhairing process leads to reduced TDS, BOD and COD in the effluent without affecting the collagen of the skin or the grain pattern [13]. It is suggested that enzymatic method can completely replace chemical method. The subsequent step is generally a combination of alkali and enzyme treatment which aims to further remove hair, grease, non-collagen component and some muscular tissue. Valeika et al. [14] investigated the action of enzymes on hair and collagen in alkaline solution. It is found that the increase of alkali is beneficial to hair removal whilst the enzyme (NUE 0.6 MPX or Biosinteze), with barely effect on the hair has a greater effect on the skin protein. In other cases, acid-enzyme treatment can be used to disintegrate the skin fibrous network isolation and purification of collagen [15]. Ju et al. [16] succeeded in preparing collagen with complete structure and uniform size distribution from bovine tendons by acid-enzyme binding method. Zhang et al. $[17,18]$ reported that collagen extracted by acid-enzlymatic methods can be used to prepare skin collagen/poly(L-lactide) fibrous membrane and scaffolds for biomedical application. Other methods have also been developed to facilitate the processes. Prasertsung et al. [19] prepared the SC using a periodic pressurized technique which was proved to be able to shorten the enzymatic treatment time and promote the removing of cells. Although the collagen fibrous structure of SC can be preserved after series of treatments as shown in Fig. 1b, its properties would be affected to a certain degree. Reing et al. [20] investigated the effects of processing methods on mechanical and biologic properties on SC. It is found that alkaline treatment decreased the growth factor, glycosaminoglycan content, the mechanical strength, and the ability to support in vitro cell growth. Particularly, the immunogenic properties of SC must be considered for applications in non-medical fileds. Protease treatment and cross-linking methods can be conducted to suppress the antigenicity of SC to some extent by either removing, shield or modify antigenic determinants $[21,22]$.

\section{Structure of SC}

Type I collage accounts for $80 \% \sim 85 \%$ of the protein in the skin. Hence, in this review, the term "collagen" will always refer to type I collagen. It is recognized that three 


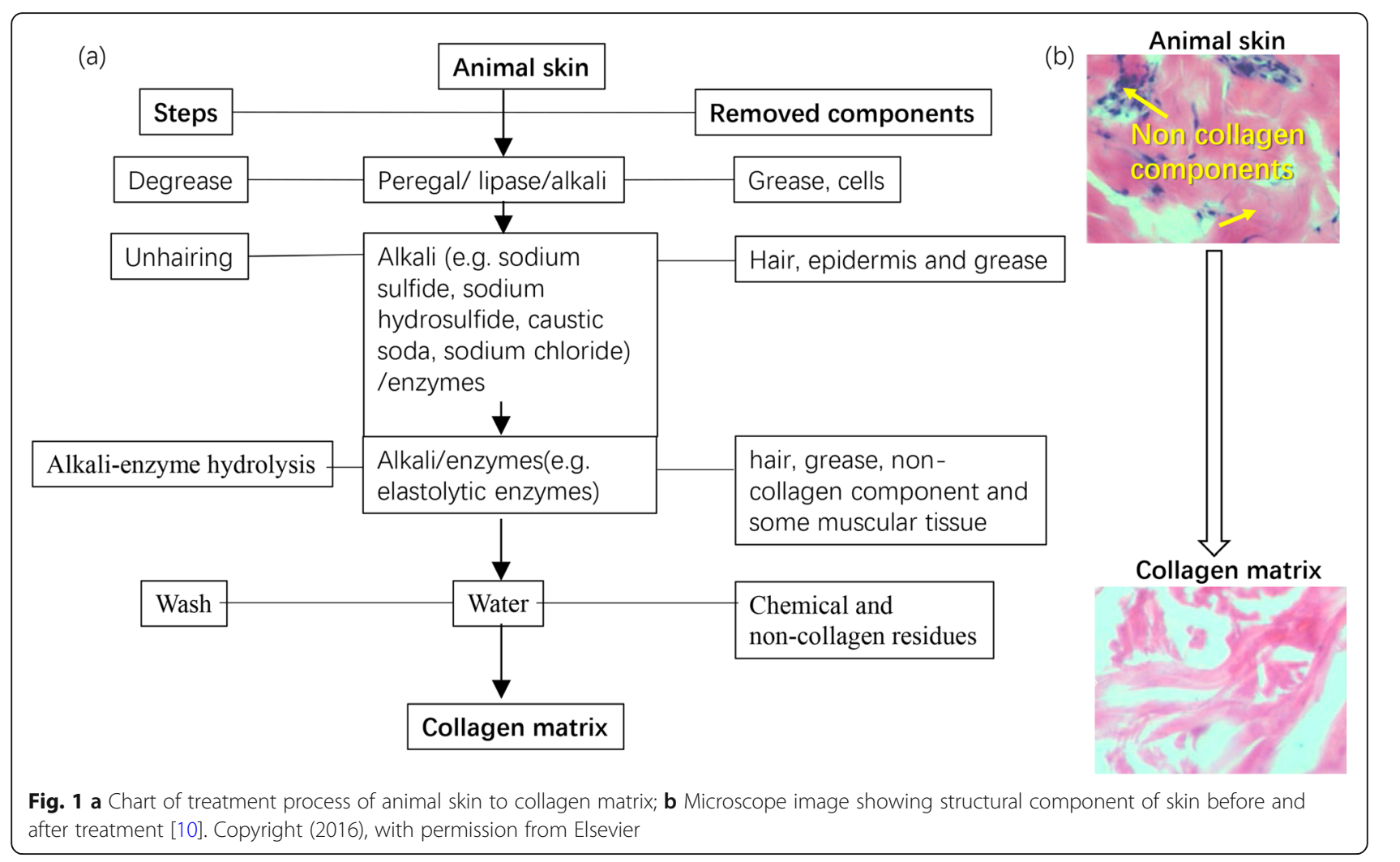

$\alpha$ chains with a distinguish sequence (Gly-X-Y) n ( $\mathrm{n}$ is approximately $350, \mathrm{X}$ is proline, $\mathrm{Y}$ is hydroxyproline) initially combine to form a three-stranded triple helix. The triple helix with a form of rod about $300 \mathrm{~nm}$ long and a dimeter of $1.5 \mathrm{~nm}$ is the monomeric unit on which the polymeric fibrous structure is built [23, 24]. Hydrogen bonds plays important roles in stable the triple helix structure [25]. Roman et al. [26] showed that the interstrand $\mathrm{H}$ bonds are significantly more important for the stability of the collagen triple helix while the ring puckering is less important for the stability of collagen. By introducing the (4S)-Aminoproline in collagen molecular chain, Siebler et al. reported that the formation of collagen triple helices can be controlled by a transannular $\mathrm{H}$ bond which is sensitive to $\mathrm{pH}$ [27]. At each end of the triple helices there are telopeptide regions. In this region, covalent bonds exist to hold the triple helix together [28-30]. Additionally, the ionic bonds and hydrophobic bonds also contribute to the construction of collagen macromolecular [31, 32]. In a research work conducted by Fallas et al. [33], a significant rearrangement of the side chain conformation allowing for packing interactions between adjacent helices was detected by $\mathrm{x}$-ray crystallography and nuclear magnetic resonance spectroscopy, which suggests that charged amino acids may play a dual role in collagen stabilization and folding. Although aromatic residues are relatively rare within the collagen triple helix, Kar et al. [34] found that they greatly accelerate the kinetics of self-association, decreasing the lag time and leading to higher-order structure of collagen.

To form the fiber, triple helices are firstly staggered to adjacent microfibrils by about $67 \mathrm{~nm}$ as a unit referred to ' $D$ '. These overlap and gap regions are retained as microfibrils assemble into fibrils. In most researches, such ' $D$ ' structure which are viewable under electron microscopy (as shown in Fig. 2) is characteristic feature of collagen [23, 35-37]. The fibrils continue to grow to form elementary fibers and subsequent fibril bundles which come together to create fibers with a wide range of fiber diameters. The fibers then characteristically intertwine with each other throughput the corium structure. Such formed fiber network is of great relevance to the physical properties of skin [38].

\section{Modification of SC}

Collagen is a scleroprotein, consisting like all proteins of amino acids linked by - $\mathrm{CO}-\mathrm{NH}$ - groups to form peptide chains. The content of acidic and basic amino acids, amino acids containing - $\mathrm{OH}$ groups is decisive for the reactivity of the collagen, which plays essential role for its functionalization $[39,40]$. The agents used to modify the SC are summarized in Table 1. Shi's group [41-44, 62-64, 74] has been conducted extensive research on realize the multi-functioned SC by modifying, such as the immobilization of tannins or metal onto SC. The 


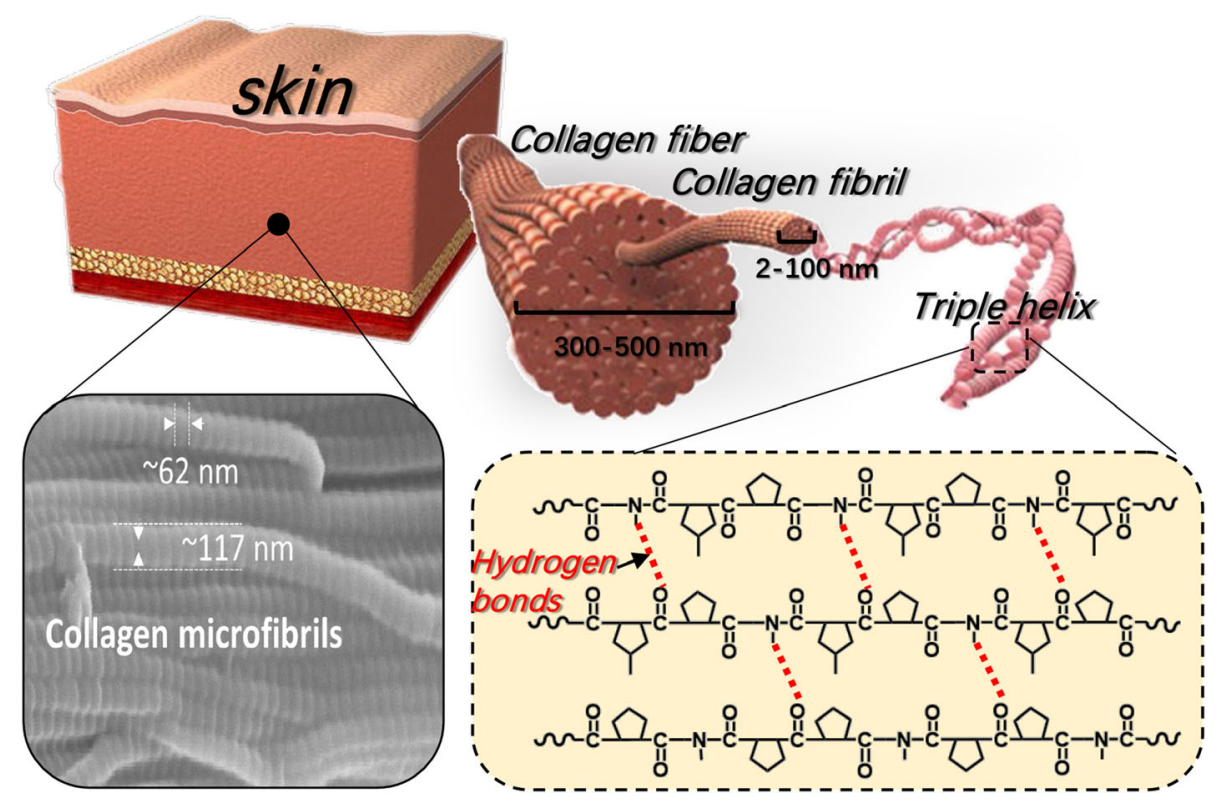

Fig. 2 Hierarchical structure of SC from triple helix to fiber network

reactive group on collagen can be combined with the hydroxyl group on the polyphenol through hydrogen bonds, and at the same time, the metal ion can also chelate both with the polyphenol and collagen. By this way, the organic/inorganic-SC system can be established, which leading to the improved properties of SC such as wave absorbing ability, radiation protection, antibacterial properties, et al. [45, 46, 62]. Other organic agents including carbodiimide, aldehydes, diisocyanates et al. has also been used to modify SC $[40,53,54,57,60]$. These agents can be covalently bonded with collagen, thus to increasing the crosslinking degree of SC. Such modified SC usually exhibites outstanding stability, mechanical property and biological performance [54, 57, 60]. Additionally, due to the hollow fearures of SC, other polymer network can be introduced in the fibrous network of SC. Through the synergy of the multi-networks, the prepared composites can show excellent properties. Han et al. [68] prepared a SC/polyurethane composite with interpenetrating duel-network. This composite is found to possess water sensitive shape memory ability. Similarly, fillers can be also incorporated into the fibrous structure of SC since the particles is able to load into the pores/capillaries. Su et al. [72] successfully modified the type I collagen by using palygorskite as a reinforcing agent which can preserve the triple helix structure of collagen. Generally, the degree of modification of SC depends on the concentration of reagents and processing time on the one hand, and on the other hand, it is also related to the number of reactive groups of SC. One way to increase the reactivity of $\mathrm{SC}$ is by releasing the reactive groups such as $-\mathrm{OH}$ and - $\mathrm{NH} 3$ during the preparation of SC through chemical and physical treatment.

\section{Advanced properties and non-medical applications}

\subsection{Water treatment}

Due to its highly reactivity to tannins, SC are utilized to prepared natural membrane with immobilized tannins for adsorption metal ions in solution [43, 75]. Because

Table 1 Modification agents for SC

\begin{tabular}{llll}
\hline Agents & \multicolumn{1}{c}{ Reactive sites } & Bond type & Reference \\
\hline Metallic salts & Carboxyl/hydroxyl groups & Complex bonds & {$[39,41-52]$} \\
Carbodiimide & Carboxyl/hydroxyl groups & Complex bonds & {$[40,53-56]$} \\
Aldehydes & Carboxyl/hydroxyl groups & Covalent bonds & {$[57-59]$} \\
Diisocyanates & Hydroxyl groups & Covalent bonds & {$[59-61]$} \\
Tannins/Phenolic agents & Peptides & Hydrogen bonds & {$[62-67]$} \\
Polyurethane & Carboxyl/hydroxyl groups/Peptides & Hydrophobic, "van der Waals" bonds, Hydrogen bonds \\
Small size substances & Pores/capillaries & H68-71] & {$[68,72,73]$} \\
\hline
\end{tabular}


vegetable tannins have multiple adjacent phenolic hydroxyls and exhibit specific affinity to metal ions, they promise to be a versatile agent for the treatment of metal ions containing wastewater. However, vegetable tannins are water-soluble compounds, which restricts their practical application. Fortunately, collagen fiber shows excellent affinity to tannis. Hence, attempts had been made to immobilize tannins onto collagen matrices. Particularly, the $\mathrm{SC}$ with fibrious structure is a promising matrix which can load tannins and improve absorption efficiency of metal ion (Fig. 3a). SEM shows that tannis has little influence on fibrious structure of collagen (Fig. 3b). Liao et al. [50] used SC to prepare a novel adsorption membrane by immobilizing with condensed vegetable tannins. The obtained membranes possess excellent adsorption and desorption characteristics for $\mathrm{UO} 2+$, as well as proper physical properties. Later.
A novel adsorbent was prepared by immobilizing barberry tannin onto skin collagen fiber (BTICF). The BTICF shows high adsorption capacity to $\mathrm{Hg}(\mathrm{II})$ in a wide $\mathrm{pH}$ range of 4.0-9.0, and a maximum adsorption capacity $(198.49 \mathrm{mg} / \mathrm{g}$ ) was reached at $\mathrm{pH} 7.0$ and $303 \mathrm{~K}$ when the initial concentration of $\mathrm{Hg}$ (II) was $200.0 \mathrm{mg} / \mathrm{L}$ (Fig. 3c). They also found that tannins immobilized collagen fiber matrixes ere effective adsorbents in the recovery of $\mathrm{Au}$ (III) from aqueous solutions. Particularly, bayberry tannins have a higher reaction activity with $\mathrm{Au}$ (III) due to the fact that the $B$ ring of bayberry tannins is a pyrogallol structure whereas the B ring of larch tannins is a catechol structure. Hence, the immobilized bayberry tannins show a higher adsorption capacity for $\mathrm{Au}(\mathrm{III})$ than immobilized larch tannins [51]. Besides, the adsorption of other metal ions including platinum(IV) and palladium(II) on bayberry tannin immobilized collagen fiber (BTICF) membrane was

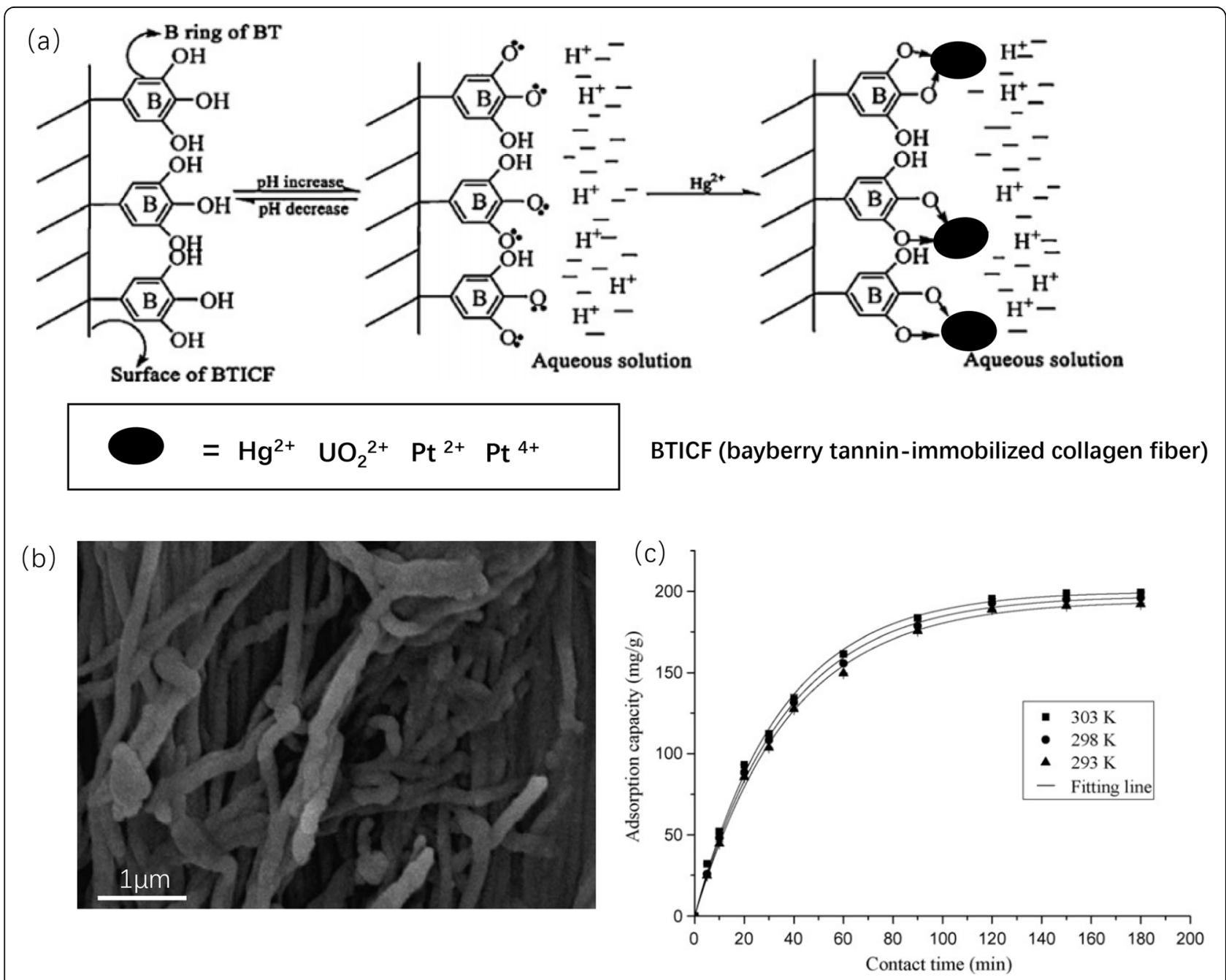

Fig. 3 a Mechanism of bayberry tannin-immobilized collagen fiber (BTICF) to metal ions; b SEM images of BTICF; c absorption capacity of modified skin collagen fiber to Hg2+ ions [43, 50]. Copyright (2009), with permission from Elsevier, and Copyright (2004), with permission from Elsevier 
investigated [75]. Results showed that the membrane exhibits ability of selective adsorption to $\mathrm{Pt}(\mathrm{IV})$ and $\mathrm{Pd}(\mathrm{II})$ in the mixture solutions of metal ions.

\subsection{Biotemplate}

Collagen fiber which contains abundant functional groups, like $-\mathrm{OH},-\mathrm{COOH}$, and $-\mathrm{NH}$, is ready to react with some metal ions, such as $\mathrm{Cr}(\mathrm{III}), \mathrm{Al}(\mathrm{III}), \mathrm{Zr}(\mathrm{IV})$, $\mathrm{Ti}(\mathrm{IV})$, and so forth. That means the metal fibers can be generated via the reaction between metal ion and collagen fiber, which lead SC to be an excellent matrix with metal ion affinity. To increase availability of reaction site, tannis with polyphenol structure can be incorporated with collagen as shown in Fig. 4a. Wu et al. [64] use SC fiber as a natural polymeric support to synthesize a novel palladium (Pd) nanoparticle catalyst. To achieve a stable immobilization of Pd on SC support, they used epigallocatechin-3-gallate (EGCG) to graft onto SC surface, acting both as dispersing and stabilizing agent for Pd nanoparticles. This nanocatalyst presented high activity and selectivity for the liquid hydrogenation of allyl alcohol and retained the catalytic efficiency after recycling for five times. Later, Wu et al. [63] further used this method to prepare size-controlled gold nanoparticles (AuNPs) supported on SC which was successfully applied as catalyst for 4-nitrophenol reduction.

Moreover, SC has unique microstructure with regular arrangements. That means the metal ions which attached to SC can grows into a fiber form in a way of collagen assembled. Thus, the obtained metal fiber can copy the structure of SC (Fig. 4b). Hence, SC is regarded as a promising biotemplate for preparing metal nanofibers with hierarchical structure. On the basis of this idea, Deng et al. [42] synthesized the alumina fiber by reaction between $\mathrm{Al}(\mathrm{III})$ and collagen fiber from animal skin which was first stabilized by vegetable tannis to enhance its fixing capacity to $\mathrm{Al}(\mathrm{III})$. In their research, hierarchical alumina fiber with ordered mesoporous distribution was successfully prepared. This metal fiber showed their improve abilities to be used as catalyst or catalyst support with high activity and selectivity owing to its high surface area and shape-selective properties [42, 44, 76]. Base on this method, hierarchical mesoporous $\mathrm{ZrO} 2$ fiber and $\mathrm{TiO} 2$ fiber were also successfully synthesized by using collagen fiber as template as shown in Fig. 4 [44, 52]. Wang et al. [47] using by using skin collagen fiber as biotemplate to prepare a sandwichstructured carbon nanofiber@SnO2@carbon. The hierarchical architecture of the C@SnO2@C nanofiber bundle guaranteed a good match between the electron transport kinetics and the $\mathrm{Li}$ + diffusion kinetics, thus realizing efficient ambipolar diffusion. This method has also been used to prepare carbon nanofiber originated from SC loaded with $\mathrm{NiFe} 2 \mathrm{O} 4$ and $\mathrm{Fe} 3 \mathrm{O} 4$ for battery application with enhanced cycling stability and rate capability [41, 49].

\subsection{Wave-adsorbing/shielding}

Researches have proved that the metal nanoparticle loaded SC has microwave absorption ability. Guo et al.

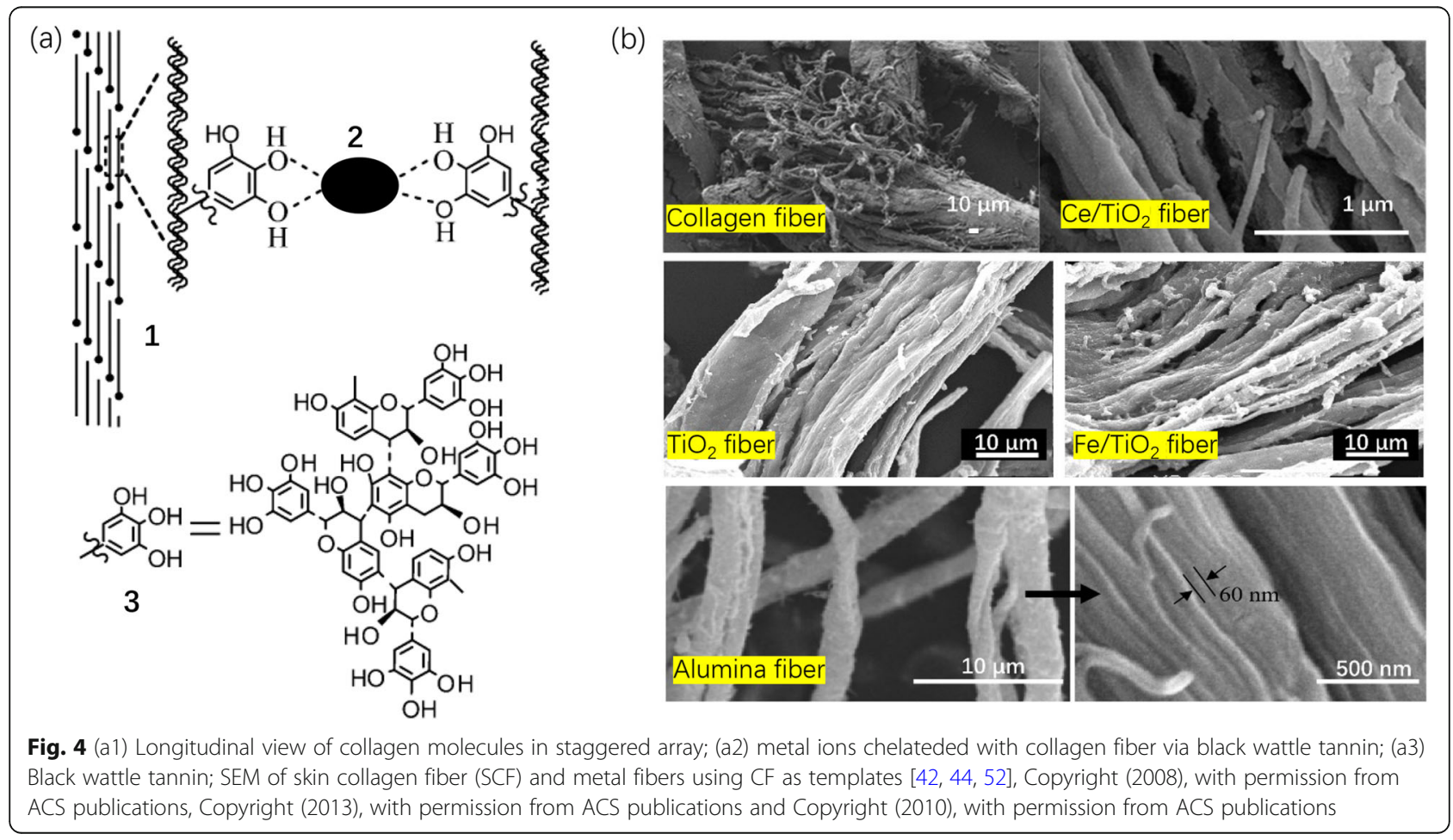


$[62,74]$ designed and fabricated the controllable Ag@Ni core-shell NPs assembled in the hierarchical intertexture of SC by a simple one-step route. This modified SC exhibited excellent microwave absorption performance in the whole $\mathrm{X}$-band, $\mathrm{C}$-band and some part of the $\mathrm{S}$ band due to its multiple defective site polarization and interfacial polarization, as well as significant eddy current effect and anisotropic energy for the microwave energy dissipation (Fig. 5a). Similarly, Wang et al. prepared the composites based on skin collagen fiber (CF) with formation of the $\mathrm{Ni}-\mathrm{Fe}-\mathrm{P}$ coating on surface. They proved that CF@Ni-Fe-P composites exhibited excellent magnetic characteristics with high saturation magnetization and low coercivity values [48]. Later, they fabricated 3D ferromagnetic hierarchical carbon nanofiber bundles (FHCNBs) using natural collagen fibers as the biotemplate, in which ferromagnetic nanoaprticles (NPs) are embedded on the HCNBs with high dispersity. Due to the induced-multiple reflection and scattering effect of FHCNBs, microwave attenuation capacity of the ferromagnetic NPs exposed on the FHCNB surface was largely improved. This method provides a new pathway for the rational realization of truly lightweight and highperformance microwave absorption materials [45]. Additionally, Liu et al. [77] fabricated a lightweight and high-performance electromagnetic waves shielding composite by coating metal nanoparticles onto leather which is made from SC (Fig. 5b). This is the first report on wave-adsorbing/shielding SC in practical supplication.

\subsection{Shape memory}

It is known that a functional material could be defined as being prepared from a "target- motivated" approach, that is, all its properties are adjusted and optimized to serve a specific purpose [78], while the smart materials are defined as materials that would either change their shape or properties between different physical domains in a useful manner under the influence of certain stimuli from the environment [79]. The research conducted by Hu's group proves that $\mathrm{SC}$ with is also belongs to field of smart materials. For the first time, it is found that $\mathrm{SC}$ has intrinsic water adaptive ability through cleavage and reforming of abundant hydrogen bonds (H-bonds) within peptide chains $[80,81]$.This kind of behavior is similarly to mechanism of some smart materials, such as shape memory materials of which the existence of reversible bonds plays an essential role [82]. Generally, mechanism governing shape memory effect of polymers can be explained by a netpoint-switch model where netpoint determines permanent shape whilst reversible bonds act as switches to allow temporary shape change [82]. In this case, collagen-based material possesses native "switch" element for realizing shape memory ability. Their research for the first time reported the shape memory performance of SC (Fig. 6a). Accordingly, the shape memory model of SC is established (Fig. 6b).

Within collagen fibers, the covalent bonds exist which can take the responsibility to hold the triple helix together. In this case, covalent bonds can work as "netpoint" for shape recovery. Considering the fact that content of covalent bonds in collagen is less, it is assumed that by introducing suitable cross linkages as "netpoint" into collagen matrix, higher shape recovery ratio after stretching deformation can be achieved. Inspired from the structure of metalloprotein, Hu's group designed an organic-metal biosystem of chromium (III) complexed skin collagen matrix (Cr-SC) [46]. As illustrated in Fig. 7, the obtained $\mathrm{Cr}$-SC retains inherent conformation of collagen and its fiber morphology in the skin. It is verified that coexistence of hydrogen bonds and chromium (III) complexing linkages can lead to achievement of a completely athermal water-responsive shape memory

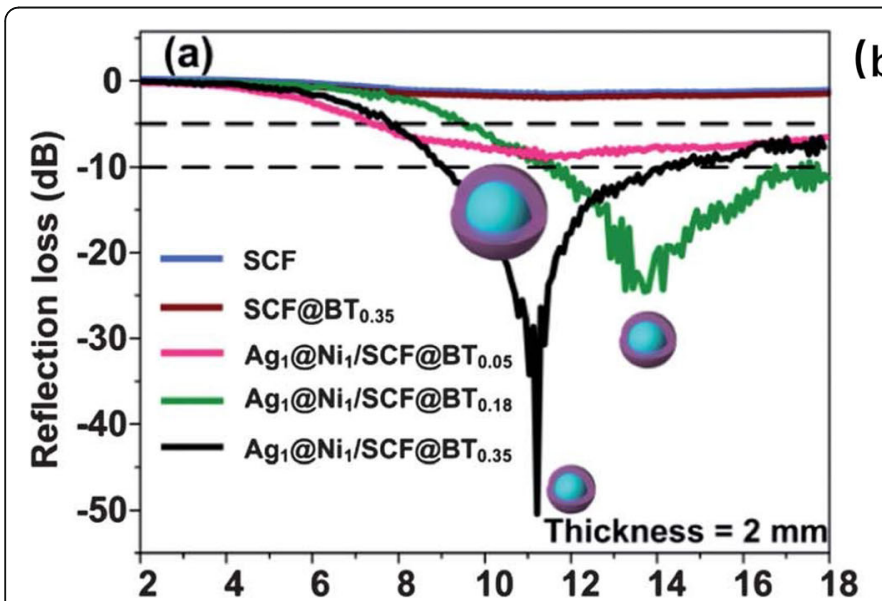

(b)

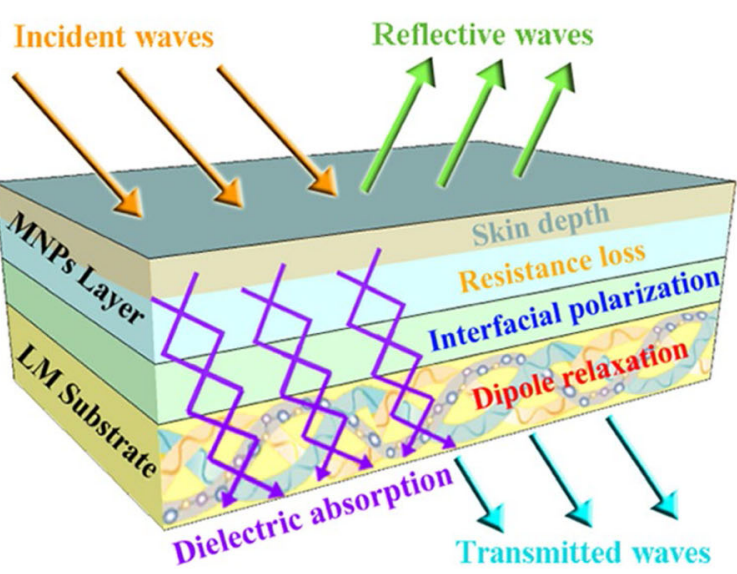

Fig. 5 a Microwave reflection losses of SCF loaded with Ag@Ni core-shell NPs [62]; b Schematic mechanism of high-performance electromagnetic waves shielding leather [77], Copyright (2018), with permission from ACS publications 


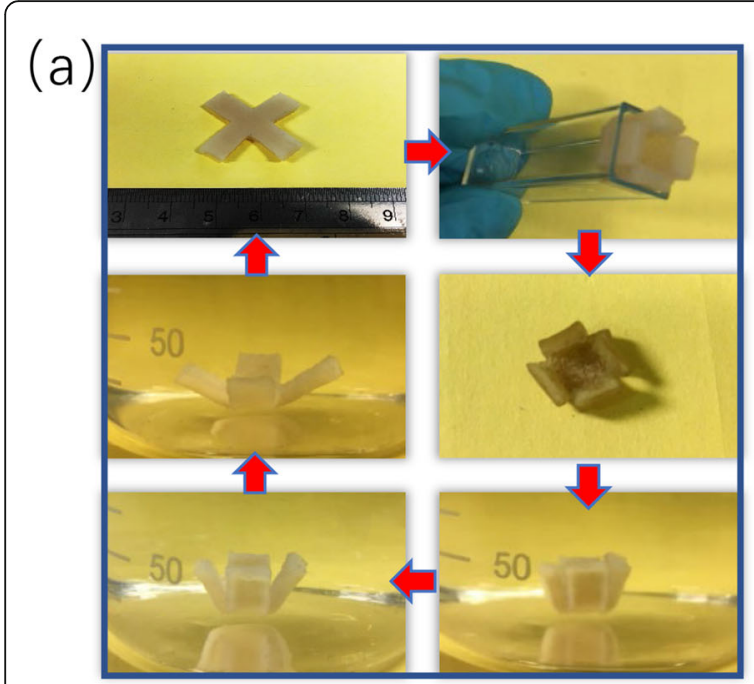

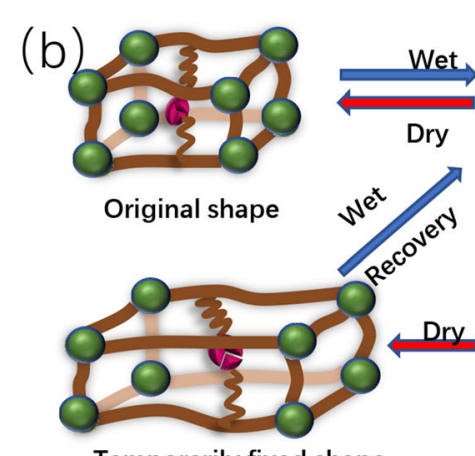

Temporarily fixed shape

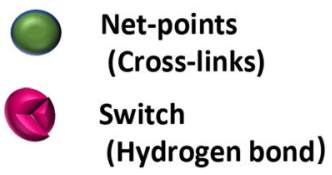

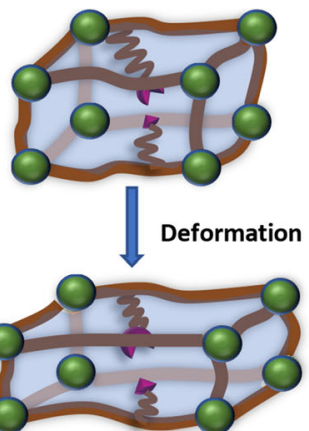

Setting shape

Spring

(Collagen peptide chain)

Water phase

Fig. 6 a Water responsive shape memory behavior of SC and $\mathbf{b}$ its mechanism model with three essential elements [81], Copyright (2018), with permission from RSC publication

ability with high shape fixation and recovery during repeatable memory cycles. Such combination of "switch" and "netpoint" facilitated water responsive shape memory ability of $\mathrm{Cr}$-SC with stretched shape fixation and recovery reaching over $80 \%$.

Moreover, SC showed good compatibility to polymer phase. Learned from the skin collagen-elastin interpenetrating network, Hu's group prepared a composite with a dual-network based on SC and polyurethane by a facile "paper-making" procedure [68]. As shown in Fig. 8, the first collagen nonwoven network was built up by spontaneous entangling and sticking of collagen fibers recycled from animal skin. Such fibrous structure was then interpenetrated by waterborne PU. By virtue of chemo-mechanical adaptability of both collagen fiber and PU elastomeric matrix, a water responsive shape memory with high shape fixation (99\%) and shape recovery $(>90 \%)$ has been achieved. Destruction and reformation of hydrogen bonds within collagen fiber works as "switch" to achieve shape deformation and fixation. This "switch" opens under the stimulation of water, while elastic entropy of PU promotes shape recovery. The obtained SC/PU composite can be degradable in simulated body fluid whilst biological evaluation in vitro (MTT assay) proved that SC/PU composite has better biocompatibility than pure PU.

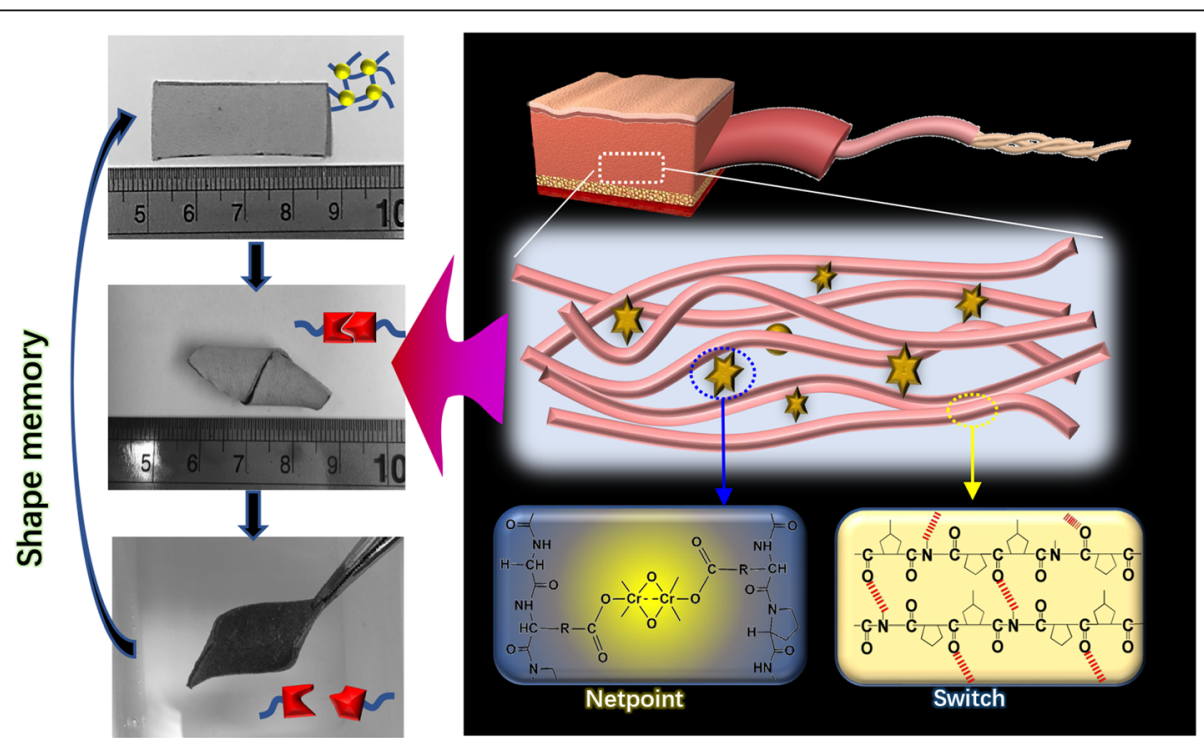

Fig. 7 Schematic of chromium (III) complexed skin collagen matrix with coalesced breathability, mechanical and shape memory properties [46], Copyright (2020), with permission from Elsevier 


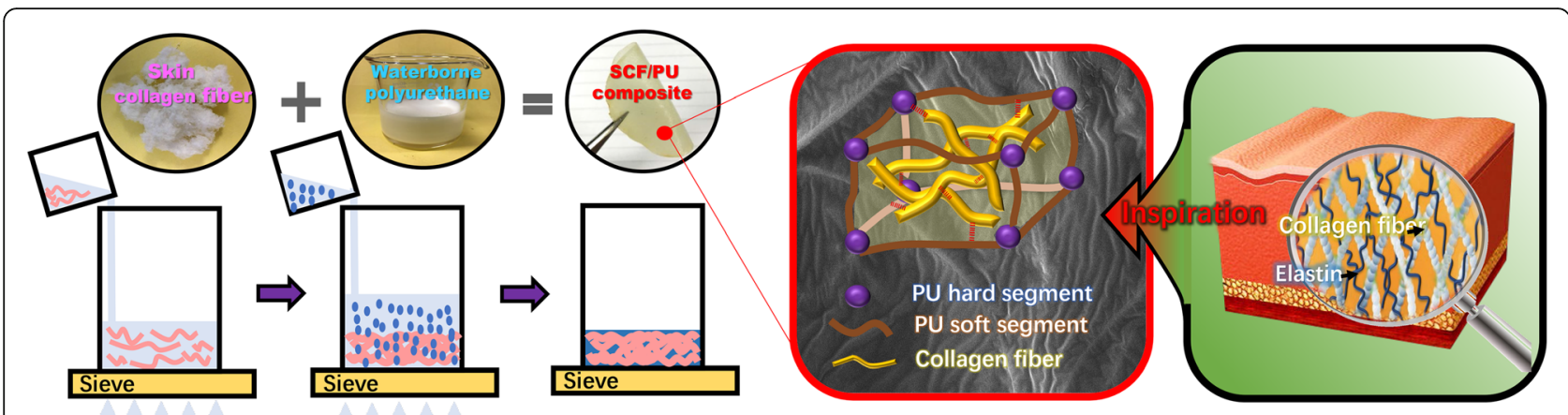

Fig. 8 facile preparation of SC/PU composite inspired from dual-network of natural skin [68], Copyright (2019), with permission from RSC publication

\subsection{Waterproof}

The SC possess the large amount of fibers with cross-scale structural deformations from microscale to nanoscale. This structure is difficult to achieve for synthetic fibers. Take advantage of SC's structural advantages, Huang et al. [83] created a durable superhydrophobic surface based on the cattle skin. Through abrasion, collagen fibers of different sizes are exposed on the surface of the skin, leading to the superhydrophobicity which can be retained even after finger-wipe, hammer-punch, screwdriver-scratch, knifecut and sandpaper abrasion due to its immediate and insitu self-repair to the damaged roughness. The author emphasized the importance of surface roughness to the such superhydrophobicity. Nevertheless, it is suggested that the fundamental hydrophobicity of collagen should be considered. Additionally, the closely arranged collagen fibers which could prevent the entrance of poly-water molecules by the limited pore size may also give a contribution. The revealed mechanisms can provide guidance to prepare the artificial superhydrophobic fabrics.

\subsection{Electronic skin}

Currently, most electronic skins are made of polymer materials. In terms of comfort, polymer membranes have poor air permeability. In comparison, substrates based on SC, such as leather possess excellent permeability. Its advantages on preparation of electronic skins has been gradually recognized [84-88]. Li et al. [86] develop a breathable, humidity-ultrastable and degradable sensory skin by using SC from cow. Such electronic skins performed superhydrophobic, unaffected sensory capability under extremely humid conditions $\left(50{ }^{\circ} \mathrm{C}\right.$ and relative humidity of $82 \%$ ) with exceptional breathability (1087 g $\mathrm{m}-2 \mathrm{~d}-1$ ). Additionally, electronic skins based on SC is environmentally friendly dur to its fully degradability. Zou et al. [84] reports a simple and designable leather based electronic skins by merging leather with nanomaterials including carbon nanotubes and silver nanowires. The general schematic of design principle is illustrated in Fig. 9. This electronic skin can be applied in flexible pressure sensors, displays, user-interactive devices, etc.

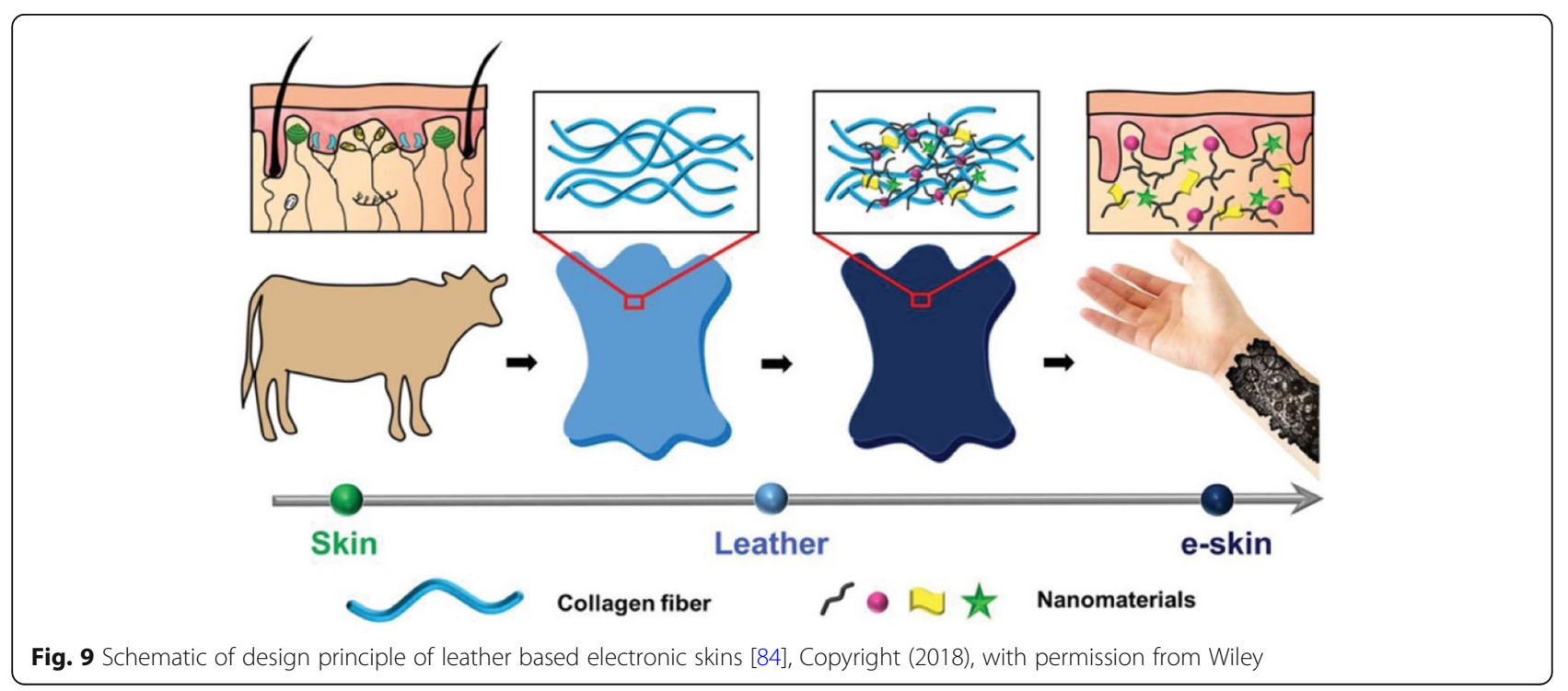


Similarly Xie et al. [85] directly used leather as a substrate to fabricate a strain sensor with leather-like microstructure by absorbing 0D conductive nanomaterials. The as-prepared sensor inherits the air permeability, mechanical property, biocompatibility and linearity performance as well as good durability. Such a sensor can be applicable to human motion monitoring and shape perception of robotic arm.

\section{Perspectives}

Due to its unique structure, SC exhibit outstanding chemical, mechanical, biological properties which has significantly benefited the development of functional materials, which is incomparable to synthetic polymers. According to the current development and based on our own understanding, perspectives are given for future development of SC.

1) Preparation methods. To date, $\mathrm{SC}$ with controllable thickness and size can be achieved by general alkalienzyme methods. Although the existing strategies provide facial methods to prepare SC, the structure of collagen fiber can also be influenced with different using of chemical, which would weak its natural properties. Therefore, more efforts can be paid on how to obtain $\mathrm{SC}$ without broke its natural structure. Additionally, the main form of SC products is limited in membranes. Hence, future challenges also include how to increase product diversity of SC.

2) Structure functions. Although the molecule structure of collagen has been widely studied, the functions, especially its intrinsic smart behavior of $\mathrm{SC}$ in materials point of view, are rarely explored. Hence, more propertied and smart function of collagen fiber regarding to its hierarchical structure from micro to macro can be investigated. For example, environmental (e.g. pH, thermo, magnetic, etc.) stimulus response of $\mathrm{SC}$ can be intensively studied for their potential application.

3) Multi-functions and high performance. The everincreasing demand for novel materials asks for multi-functions and high performance. As the biomass with high reactivity, SC has a lot of room for chemical and physical modification to enhance its performance. For example, by chemical modification and or compositing technology, the SC or its composites would exhibit enhanced property due to the synergistic effect resulted from natural collagen fiber and polymers.

4) Extended applications. Traditionally, the application of SC is focused on biological application as such wound dressing and scaffold. Up to date, some other potential application including metal and microwave absorption have been reported, however, the complex and high cost preparation process limited its practical development. Recently, the shape memory performance of SC has been revealed, indicating the application of SC as smart device which deserving further exploring. As major component of skin, SC should have advantages when applied in fields such as artificial skin. On the other hand, back to its fiber form, 3D fibrous network of SC could have broad range of application as covering materials to provide both strength, flexibility, protectiveness, permeability, etc.

\section{Acknowledgements}

This work was financially supported by the National Natural Science Foundation of China (51673162), the Hong Kong General Research Fund (15201719; 152098/15E), Research Grants Council, University Grants Committee (PolyU 5158/13E), and Hong Kong PhD Fellowship (PF15-15110).

Authors' contributions

Yanting Han: Writing- Original draft preparation. Jinlian Hu: Supervision, Writing, Reviewing and Editing. Gang Sun: Reviewing and Editing. The author(s) read and approved the final manuscript.

Authors' information

Not applicable.

Funding

National Natural Science Foundation of China (51673162), the Hong Kong General Research Fund (15201719; 152098/15E), Research Grants Council, University Grants Committee (PolyU 5158/13E), and Hong Kong PhD Fellowship (PF15-15110).

Availability of data and materials Not applicable.

\section{Competing interests}

The authors declare no conflict of interest.

\section{Author details}

${ }^{1}$ Institute of Textiles and Clothing, the Hong Kong Polytechnic University, Hong Kong, China. ${ }^{2}$ Department of Biomedical Engineering, City University of Hong Kong, Kowloon, Hong Kong, China. ${ }^{3}$ Division of Textiles and Clothing, University of California, Davis, Davis, USA.

Received: 20 April 2020 Accepted: 18 December 2020

Published online: 15 March 2021

\section{References}

1. Eisenberg $D$. The discovery of the a-helix and $\beta$-sheet, the principal structural features of proteins. Proc Natl Acad Sci. 2003;100(20):11207-10.

2. Terzi A, Storelli E, Bettini S, Sibillano T, Altamura D, Salvatore L, et al. Effects of processing on structural, mechanical and biological properties of collagen-based substrates for regenerative medicine. Sci Rep. 2018;8(1):1429.

3. Chang S-W, Buehler MJ. Molecular biomechanics of collagen molecules. Mater Today. 2014;17(2):70-6.

4. Veres SP, Harrison JM, Lee JM. Mechanically overloading collagen fibrils uncoils collagen molecules, placing them in a stable, denatured state. Matrix Biol. 2014;33:54-9.

5. Yu B, Kang S-Y, Akthakul A, Ramadurai N, Pilkenton M, Patel A, et al. An elastic second skin. Nat Mater. 2016;15(8):911.

6. Chen J-P, Chang G-Y, Chen J-K. Electrospun collagen/chitosan nanofibrous membrane as wound dressing. Colloids Surf A Physicochem Eng Asp. 2008; 313:183-8.

7. Li M, Mondrinos MJ, Gandhi MR, Ko FK, Weiss AS, Lelkes PI. Electrospun protein fibers as matrices for tissue engineering. Biomaterials. 2005;26(30): 5999-6008. 
8. Zeng J, Xu X, Chen X, Liang Q, Bian X, Yang L, et al. Biodegradable electrospun fibers for drug delivery. J Control Release. 2003;92(3):227-31.

9. Montagna W. The structure and function of skin: Elsevier; 2012.

10. Liu X, Dan N, Dan W. Preparation and characterization of an advanced collagen aggregate from porcine acellular dermal matrix. Int J Biol Macromol. 2016:88:179-88.

11. Takami H, Nakamura S, Aono R, Horikoshi K. Degradation of human hair by a thermostable alkaline protease from alkaliphilic Bacillus sp. no. AH-101. Biosci Biotechnol Biochem. 1992;56(10):1667-9.

12. Dettmer A, Cavalli É, Ayub MA, Gutterres M. Environmentally friendly hide unhairing: enzymatic hide processing for the replacement of sodium sulfide and delimig. J Clean Prod. 2013;47:11-8

13. Rose C, Suguna L, Rajini R, Samivelu N, Rathinasamy V, Ramalingam S, et al. Process for lime and sulfide free unhairing of skins or hides using animal and/or plant enzymes. Google Patents; 2007.

14. Valeika V, Beleška K, Valeikienè V, Kolodzeiskis V. An approach to cleaner production: from hair burning to hair saving using a lime-free unhairing system. J Clean Prod. 2009;17(2):214-21.

15. Dan W, Chen Y, Dan N, Zheng X, Wang L, Yang C, et al. Multi-level collagen aggregates and their applications in biomedical applications. Int J Polym Anal Charact. 2019;24(8):667-83.

16. Ju H-Y, Liu M, Dan W-H, Hu Y, Lin H, Dan N-H. Dynamic rheological property of type I collagen fibrils. J Mechanics Med Biol. 2013;13(06): 1340015.

17. Zhang M, Wang J, Xu W, Luan J, Li X, Zhang Y, et al. The mechanical property of Rana chensinensis skin collagen/poly (L-lactide) fibrous membrane. Mater Lett. 2015;139:467-70.

18. Zhang $M, L i Z$, Jiang $P$, Lin $T$, Li X, Sun D. Characterization and cell response of electrospun $R$ ana chensinensis skin collagen/poly (I-lactide) scaffolds with different fiber orientations. J Appl Polym Sci. 2017;134(34):45109.

19. Prasertsung I, Kanokpanont S, Bunaprasert T, Thanakit V, Damrongsakkul S. Development of acellular dermis from porcine skin using periodic pressurized technique. J Biomed Mater Res B Appl Biomater. 2008;85(1):210-9.

20. Reing JE, Brown BN, Daly KA, Freund JM, Gilbert TW, Hsiong SX, et al. The effects of processing methods upon mechanical and biologic properties of porcine dermal extracellular matrix scaffolds. Biomaterials. 2010;31(33):8626-33.

21. Lynn A, Yannas I, Bonfield W. Antigenicity and immunogenicity of collagen. J Biomed Mater Res B Appl Biomater. 2004;71 (2):343-54.

22. Chevallay B, Herbage D. Collagen-based biomaterials as 3D scaffold for cell cultures: applications for tissue engineering and gene therapy. Med Biol Eng Comput. 2000;38(2):211-8.

23. Brodsky B, Ramshaw JA. The collagen triple-helix structure. Matrix Biol. 1997; 15(8-9):545-54.

24. Kramer RZ, Bella J, Mayville P, Brodsky B, Berman HM. Sequence dependent conformational variations of collagen triple-helical structure. Nat Struct Mol Biol. 1999:6(5):454

25. Shoulders MD, Raines RT. Collagen structure and stability. Annu Rev Biochem. 2009:78:929-58,

26. Erdmann $\mathrm{RS}$, Wennemers $\mathrm{H}$. Importance of ring puckering versus interstrand hydrogen bonds for the conformational stability of collagen. Angew Chem. 2011;123(30):6967-70.

27. Siebler C, Erdmann RS, Wennemers H. Switchable proline derivatives: tuning the conformational stability of the collagen triple helix by $\mathrm{pH}$ changes. Angew Chem. 2014;126(39):10508-12.

28. Eyre DR, Paz MA, Gallop PM. Cross-linking in collagen and elastin. Annu Rev Biochem. 1984;53(1):717-48.

29. Kwansa AL, De Vita R, Freeman JW. Mechanical recruitment of N-and Ccrosslinks in collagen type I. Matrix Biol. 2014;34:161-9.

30. Tanzer ML. Cross-linking of collagen. Science. 1973;180(4086):561-6.

31. Bella J, Berman HM. Crystallographic evidence for $\mathrm{Ca}-\mathrm{H} \cdots \mathrm{O}=\mathrm{C}$ hydrogen bonds in a collagen triple helix. J Mol Biol. 1996;264(4):734-42.

32. Ramachandran $\mathrm{G}$, Chandrasekharan R. Interchain hydrogen bonds via bound water molecules in the collagen triple helix. Biopolymers. 1968;6(11): 1649-58.

33. Fallas JA, Dong J, Tao YJ, Hartgerink JD. Structural insights into charge pair interactions in triple helical collagen-like proteins. J Biol Chem. 2012;287(11): 8039-47.

34. Kar K, Ibrar S, Nanda V, Getz TM, Kunapuli SP, Brodsky B. Aromatic interactions promote self-association of collagen triple-helical peptides to higher-order structures. Biochemistry. 2009;48(33):7959-68.
35. Birk DE, Bruckner P. Collagen suprastructures. Collagen: Springer; 2005. p. 185-205.

36. Brodsky B, Persikov AV. Molecular structure of the collagen triple helix. Advances in protein chemistry. 70: Elsevier; 2005. p. 301-39.

37. Smith J. Molecular pattern in native collagen. Nature. 1968;219(5150):157-8.

38. Zimmermann EA, Gludovatz B, Schaible E, Dave NK, Yang W, Meyers MA, et al. Mechanical adaptability of the Bouligand-type structure in natural dermal Armour. Nat Commun. 2013:4(1):1-7.

39. Gustavson K. The effect of esterification of the carboxyl groups of collagen upon its combination with chromium compounds. J Am Chem Soc. 1952; 74(18):4608-11.

40. Everaerts F, Torrianni M, Hendriks M, Feijen J. Quantification of carboxyl groups in carbodiimide cross-linked collagen sponges. J Biomed Mater Res A. 2007;83(4):1176-83.

41. Chen Z, Zhou J, Wang X, Liao X, Huang X, Shi B. Natural collagen fiberenabled facile synthesis of carbon@ Fe 3 O 4 core-shell nanofiber bundles and their application as ultrahigh-rate anode materials for Li-ion batteries. RSC Adv. 2016;6(13):10824-30.

42. Deng D, Tang R, Liao X, Shi B. Using collagen fiber as a template to synthesize hierarchical mesoporous alumina fiber. Langmuir. 2008;24(2):368-70.

43. Huang $X$, Liao $X$, Shi B. Hg (II) removal from aqueous solution by bayberry tannin-immobilized collagen fiber. J Hazard Mater. 2009;170(2):1141-8.

44. Xiao G, Huang X, Liao X, Shi B. One-pot facile synthesis of cerium-doped $\mathrm{TiO} 2$ mesoporous nanofibers using collagen fiber as the biotemplate and its application in visible light photocatalysis. J Phys Chem C. 2013;117(19): 9739-46.

45. Wang X, Huang X, Chen Z, Liao X, Liu C, Shi B. Ferromagnetic hierarchical carbon nanofiber bundles derived from natural collagen fibers: truly lightweight and high-performance microwave absorption materials. J Mater Chem C. 2015;3(39):10146-53.

46. Han $Y$, Jiang $Y, H u J$, Chen $X$. Achieving coalesced breathability, mechanical and shape memory properties of collagen fibrous matrix through complexing with chromium (III). Mater Des. 2020;186:108206.

47. Wang X, Li J, Chen Z, Lei L, Liao X, Huang X, et al. Hierarchically structured C@SnO2@ C nanofiber bundles with high stability and effective ambipolar diffusion kinetics for high-performance Li-ion batteries. J Mater Chem A. 2016:4(48):18783-91.

48. Wang $X$, Liao $X$, Zhang W, Shi B. Bio-inspired fabrication of hierarchical NiFe-P coated skin collagen fibers for high-performance microwave absorption. Phys Chem Chem Phys. 2015;17(3):2113-20.

49. Chen Z, Zhang $Y$, Wang $X$, Sun W, Dou S, Huang $X$, et al. Fastpulverization enabled simultaneous enhancement on cycling stability and rate capability of C@ NiFe2O4 hierarchical fibrous bundle. J Power Sources. 2017;363:209-17.

50. Liao X, Ma H, Wang R, Shi B. Adsorption of UO22+ on tannins immobilized collagen fiber membrane. J Membr Sci. 2004;243(1):235-41.

51. Liao X, Zhang M, Shi B. Collagen-fiber-immobilized tannins and their adsorption of au (III). Ind Eng Chem Res. 2004;43(9):2222-7.

52. Cai L, Liao X, Shi B. Using collagen Fiber as a template to synthesize TiO2 and $\mathrm{Fe} \times \mathrm{TTiO} 2$ Nanofibers and their catalytic behaviors on the visible lightassisted degradation of Orange II. Ind Eng Chem Res. 2010;49(7):3194-9.

53. Bax DV, Davidenko N, Gullberg D, Hamaia SW, Farndale RW, Best SM, et al. Fundamental insight into the effect of carbodiimide crosslinking on cellular recognition of collagen-based scaffolds. Acta Biomater. 2017;49:218-34.

54. Bax DV, Davidenko N, Hamaia SW, Farndale RW, Best SM, Cameron RE. Impact of UV-and carbodiimide-based crosslinking on the integrin-binding properties of collagen-based materials. Acta Biomater. 2019;100:280-91.

55. Damink LO, Dijkstra P, Van Luyn M, Van Wachem P, Nieuwenhuis P, Feijen J. Cross-linking of dermal sheep collagen using a water-soluble carbodiimide. Biomaterials. 1996;17(8):765-73.

56. Zeeman R, Dijkstra PJ, van Wachem PB, van Luyn MJ, Hendriks M, Cahalan PT, et al. Successive epoxy and carbodiimide cross-linking of dermal sheep collagen. Biomaterials. 1999;20(10):921-31.

57. Fathima NN, Madhan B, Rao JR, Nair BU, Ramasami T. Interaction of aldehydes with collagen: effect on thermal, enzymatic and conformational stability. Int J Biol Macromol. 2004;34(4):241-7.

58. Bowes J, Cater $C$. The interaction of aldehydes with collagen. Biochim Biophys Acta. 1968;168(2):341-52

59. Damink LO, Dijkstra P, Van Luyn M, Van Wachem P, Nieuwenhuis P, Feijen J. Crosslinking of dermal sheep collagen using hexamethylene diisocyanate. J Mater Sci Mater Med. 1995;6(7):429-34. 
60. Gooch B, Smart N, Wajed S. Transthoracic repair of an incarcerated diaphragmatic hernia using hexamethylene diisocyanate cross-linked porcine dermal collagen (Permacol). Gen Thorac Cardiovasc Surg. 2012; 60(3):145-8.

61. Smart NJ, Velineni R, Khan D, Daniels IR. Parastomal hernia repair outcomes in relation to stoma site with diisocyanate cross-linked acellular porcine dermal collagen mesh. Hernia. 2011;15(4):433-7.

62. Guo J, Wang X, Miao P, Liao X, Zhang W, Shi B. One-step seeding growth of controllable Ag@ Ni core-shell nanoparticles on skin collagen fiber with introduction of plant tannin and their application in high-performance microwave absorption. J Mater Chem. 2012;22(24):11933-42.

63. Wu H, Huang X, Gao M, Liao X, Shi B. Polyphenol-grafted collagen fiber as reductant and stabilizer for one-step synthesis of size-controlled gold nanoparticles and their catalytic application to 4-nitrophenol reduction. Green Chem. 2011;13(3):651-8.

64. Wu H, Wu C, He Q, Liao X, Shi B. Collagen fiber with surface-grafted polyphenol as a novel support for Pd (0) nanoparticles: synthesis, characterization and catalytic application. Mater Sci Eng C. 2010;30(5): 770-6.

65. Vidal CM, Leme AA, Aguiar TR, Phansalkar R, Nam J-W, Bisson J, et al. Mimicking the hierarchical functions of dentin collagen cross-links with plant derived phenols and phenolic acids. Langmuir. 2014;30(49): 14887-93.

66. Vijayaraghavan R, Thompson B, MacFarlane D, Kumar R, Surianarayanan M, Aishwarya $\mathrm{S}$, et al. Biocompatibility of choline salts as crosslinking agents for collagen based biomaterials. Chem Commun. 2009:46(2):294-6.

67. Han B, Jaurequi J, Tang BW, Nimni ME. Proanthocyanidin: a natural crosslinking reagent for stabilizing collagen matrices. J Biomed Mater Res A. 2003;65(1):118-24.

68. Han $Y, H u$ J, Chen X. A skin inspired bio-smart composite with water responsive shape memory ability. Mater Chem Front. 2019;3:1128.

69. He X, Zhai Z, Wang Y, Wu G, Zheng Z, Wang Q, et al. New method for coupling collagen on biodegradable polyurethane for biomedical application. J Appl Polym Sci. 2012;126(S1):E354-E61.

70. Caddeo S, Mattioli-Belmonte M, Cassino C, Barbani N, Dicarlo M, Gentile P, et al. Newly-designed collagen/polyurethane bioartificial blend as coating on bioactive glass-ceramics for bone tissue engineering applications. Mater Sci Eng C. 2019;96:218-33.

71. Han $Y$, Jiang $Y$, Hu J. Collagen incorporation into waterborne polyurethane improves breathability, mechanical property, and self-healing ability. Compos A: Appl Sci Manuf. 2020:105854.

72. Su D, Wang C, Cai S, Mu C, Li D, Lin W. Influence of palygorskite on the structure and thermal stability of collagen. Appl Clay Sci. 2012;62:41-6.

73. Luo Q, Nakade R, Dong X, Rong Q, Wang X. Effect of mineral-collagen interfacial behavior on the microdamage progression in bone using a probabilistic cohesive finite element model. J Mech Behav Biomed Mater. 2011:4(7):943-52.

74. Guo J, Wang X, Liao X, Zhanga W, Shi B. Skin collagen fiber-biotemplated synthesis of size-tunable silver nanoparticle-embedded hierarchical intertextures with lightweight and highly efficient microwave absorption properties. J Phys Chem C. 2012;116(14):8188-95.

75. Smeenk JM, Schön P, Otten MBJ, Speller S, Stunnenberg HG, van Hest JCM. Fibril formation by Triblock copolymers of Silklike $\beta$-sheet polypeptides and poly(ethylene glycol). Macromolecules. 2006;39(8):2989-97.

76. Qi X, Yao X, Deng S, Zhou T, Fu Q. Water-induced shape memory effect of graphene oxide reinforced polyvinyl alcohol nanocomposites. J Mater Chem A. 2014:2(7):2240-9.

77. Liu C, Wang X, Huang X, Liao X, Shi B. Absorption and reflection contributions to the high performance of electromagnetic waves shielding materials fabricated by compositing leather matrix with metal nanoparticles. ACS Appl Mater Interfaces. 2018;10(16):14036-44.

78. Thomas A. Functional materials: from hard to soft porous frameworks. Angew Chem Int Ed. 2010;49(45):8328-44

79. Khoo ZX, Teoh JEM, Liu Y, Chua CK, Yang S, An J, et al. 3D printing of smart materials: a review on recent progresses in $4 D$ printing. Virtual Phys Prototyping. 2015;10(3):103-22.

80. O'leary LE, Fallas JA, Bakota EL, Kang MK, Hartgerink JD. Multi-hierarchical self-assembly of a collagen mimetic peptide from triple helix to nanofibre and hydrogel. Nat Chem. 2011;3(10):821.

81. Han Y, Hu J, Jiang L. Collagen skin, a water-sensitive shape memory material. J Mater Chem B. 2018;6(31):5144-52.
82. Hu J, Zhu Y, Huang H, Lu J. Recent advances in shape-memory polymers: structure, mechanism, functionality, modeling and applications. Prog Polym Sci. 2012;37(12):1720-63.

83. Huang $X$, Kong $X$, Cui $Y$, Ye $X$, Wang $X$, Shi B. Durable superhydrophobic materials enabled by abrasion-triggered roughness regeneration. Chem Eng J. 2018;336:633-9.

84. Zou B, Chen Y, Liu Y, Xie R, Du Q, Zhang T, et al. Repurposed leather with sensing capabilities for multifunctional electronic skin. Adv Sci. 2019;6(3): 1801283.

85. Xie R, Hou S, Chen Y, Zhang K, Zou B, Liu Y, et al. Leather-based strain sensor with hierarchical structure for motion monitoring. Adv Mater Technol. 2019;4(10):1900442.

86. Ke L, Wang Y, Ye X, Luo W, Huang X, Shi B. Collagen-based breathable, humidity-ultrastable and degradable on-skin device. J Mater Chem C. 2019; 7(9):2548-56.

87. Xie R, Du Q, Zou B, Chen Y, Zhang K, Liu Y, et al. Wearable leather-based electronics for respiration monitoring. ACS Appl Bio Mater. 2019;2(4):1427-31.

88. Abodurexiti A, Yang C, Maimaitiyiming X. High-performance flexible pressure and temperature sensors with complex leather structure. Macromol Mater Eng. 2020;305(7):2000181.

\section{Publisher's Note}

Springer Nature remains neutral with regard to jurisdictional claims in published maps and institutional affiliations.

\section{Submit your manuscript to a SpringerOpen ${ }^{\circ}$ journal and benefit from:}

- Convenient online submission

- Rigorous peer review

- Open access: articles freely available online

High visibility within the field

- Retaining the copyright to your article

Submit your next manuscript at $>$ springeropen.com 